University of Nebraska - Lincoln

DigitalCommons@University of Nebraska - Lincoln

August 2003

\title{
Barriers to physicians' decisions to discuss hospice: Insights gained from the United States hospice model
}

\author{
E. Kiernan McGorty \\ University of Nebraska-Lincoln \\ Brian H. Bornstein \\ University of Nebraska-Lincoln, bbornstein2@unl.edu
}

Follow this and additional works at: https://digitalcommons.unl.edu/psychfacpub

Part of the Psychiatry and Psychology Commons

McGorty, E. Kiernan and Bornstein, Brian H., "Barriers to physicians' decisions to discuss hospice: Insights gained from the United States hospice model" (2003). Faculty Publications, Department of Psychology. 166.

https://digitalcommons.unl.edu/psychfacpub/166

This Article is brought to you for free and open access by the Psychology, Department of at DigitalCommons@University of Nebraska - Lincoln. It has been accepted for inclusion in Faculty Publications, Department of Psychology by an authorized administrator of DigitalCommons@University of Nebraska - Lincoln. 


\title{
Barriers to physicians' decisions to discuss hospice: Insights gained from the United States hospice model
}

\author{
E. Kiernan McGorty BS and Brian H. Bornstein PhD MLS \\ Department of Psychology, University of Nebraska-Lincoln
}

\section{Correspondence:}

Dr. Brian Bornstein, Department of Psychology, University of Nebraska-Lincoln,

238 Burnett Hall, Lincoln NE 68588-0308 USA

Accepted for publication January 10, 2003.

\begin{abstract}
:
Due to its comprehensive and cost-saving design, hospice has become a critical component of health care. Physicians have become the primary gatekeepers to information on hospice and sources of referral to hospice. However, many physicians do not discuss hospice options until late in the disease course, when patients and their families are no longer able to benefit from hospice services. Although physicians, as well as patients and hospice personnel, cite patient and hospice structure factors as barriers, the present article will focus on barriers physicians have more control over, such as their discomfort discussing terminality and fear of losing contact with patients. Focusing on the American hospice model, the present article will review past findings, examine gaps in the research, and propose systematic ways to discern whether the factors physicians claim are barriers actually affect their decision making about hospice referral.
\end{abstract}

Keywords: decision-making, hospice

\section{Introduction}

Given the rising costs of acute hospital care and the increasing number of elderly patients with terminal diseases, hospice has become and will continue to be a crucial component of end-of-life health care (Thalhuber 1995; Emanuel 1996; Weggel 1999). The concept of hospice dates back as far as Roman times, but the modern hospice movement began in Britain in the mid-1960s as a grass-roots network of volunteers committed to improving the treatment of dying patients (Carwein 1986). Today, hospices' interdisciplinary approach to palliative care is a widely accepted model of care for terminally ill patients in both Britain and the United States of America (USA). A key difference between the British and American systems is that hospice care in Britain is based principally on an interprofessional model (i.e. a variety of health professionals are involved), whereas hospice care in the USA relies more heavily on a nursing model (Carwein 1986). According to the Hospice Information Service's (2002) palliative care facts and figures, at least $60 \%$ of cancer deaths in the United Kingdom occur under inpatient or outpatient hospice care. In comparison, $42 \%$ of cancer deaths in the USA occur under hospice care (National Statistics on Death and Hospice 1994). These percentages demonstrate the scope of hospice care in both Britain and the United States.

Bonham et al. (1986), however, found that 59\% of families with terminally ill cancer patients who lived in areas served by hospices did not receive information about hospice options during the critical period when they were making terminal care decisions. Although anyone can refer patients to hospice, physicians have become the primary gatekeepers to information on hospice and sources of referral to hospice. Because physicians often do not present hospice options until late in the disease course, many patients and their families are unable to benefit from hospice services. Unfortunately, the primary reason patients are not admitted to hospice is death before admission, rather than their decision to decline hospice services (MacDonald 1989). Hospice 
care may not be appropriate for every terminally ill patient, but the aim of the health care system should be to ensure that the choice of hospice care is available to patients for whom it would be beneficial.

In the present article, we identify treating physicians as critical to hospice utilization and focus on impediments to their decisions to discuss hospice. The present article will: (i) make an argument for increased disclosure of hospice services to patients and their families; (ii) discuss the influence of physicians on the decision to use hospice; and (iii) review what factors affect physicians' decisions to discuss hospice options and when, if at all, they consider hospice discussions appropriate. In addition, suggestions will be made about systematic ways in which researchers can further investigate the barriers to physicians' decisions to discuss and utilize hospice. Although the present article's emphasis is on the American hospice model, the recommendations discussed below should be applicable to other countries that use hospice as well.

\section{The benefits of hospice}

Hospice is a desirable form of care because of its ability to meet the psychological and emotional needs of patients and their families in a cost-effective manner. A meta-analysis of studies on cost savings from hospice revealed that hospice care can save up to $40 \%$ of health care costs during the last month of life and $17 \%$ over the last 6 months of life (Emanuel 1996). Among patients with cancer, health care costs were found to be 13-20\% lower for decedents receiving hospice care as compared with those not receiving hospice care (Emanuel et al. 2002). Hospices typically carry the burden of up-front transferral costs, around $\$ 2000$, that are reimbursed by Medicare and Medicaid at a rate of around $\$ 100$ per day (Stillman $\&$ Syrjala 1999). Due to this system of third-party reimbursement, patients who die within 3 weeks of transferral do not allow hospices to recoup their initial investment.

According to American hospice regulations, patients are not appropriate for care until they have less than 6 months, or about 180 days, to live. Studies consistently show that the majority of patients admitted to hospice die well before they have received care for 180 days. Studies have found that the median survival time of hospice patients ranges from 23 to 36 days, with longer stays by patients covered by Medicare (Christakis 1994; Christakis \& Escarce 1996; Stillman \& Syrjala 1999). About $15 \%$ of patients die within a week of admission (Christakis 1994; Stillman \& Syrjala 1999), and $29 \%$ die within 2 weeks of admission (Christakis \& Escarce 1996).

Several studies have shown that physicians' predictions regarding life expectancies are often imprecise. Physicians commonly overestimate the life expectancy of their terminally ill patients (Pearlman 1988; Rhymes 1990). The 6-month regulation that penalized hospices and physicians when patients lived too long has recently been revised, so that there is now no penalty for an incorrect prognosis if the disease runs its normal course (Thalhuber 1995). Research needs to examine the direct and indirect costs of inaccurate prognoses by relating the number of days before or after expected death of hospice patients to the costs of care incurred. Brief stays under hospice care are not only financially burdensome to hospices, but they also disrupt the interdisciplinary and comprehensive design of hospice care.

Due to its compassionate and cost-saving design, hospice both supplements and enhances the traditional care provided by home care programs, transitional care units and nursing homes. Hospice offers quality end-of-life care that leads to high levels of patient and family satisfaction (Bonham et al. 1986; Rhymes 1990; Stillman \& Syrjala 1999). Although satisfaction with hospice is high regardless of the timing of patient admittance, caregivers report more satisfaction with hospice when the patient has been enrolled 30 days or more compared with less than 30 days (Stillman \& Syrjala 1999).

Besides caregivers, physicians and hospice staff also recognize the benefits of timely hospice referrals. According to Christakis (1997), hospice physicians and other personnel consider 80-90 days an optimal amount of time for hospice to provide psychological and physical support for the patient and family (as cited in Stillman \& Syrjala 1999). Patients who are late referrals might benefit from a more humane and clinically appropriate longer hospice stay. A late transfer may result in harmful discontinuity in care and a requirement of hospice nurses to provide hyperacute death care rather than palliative care. In addition, a longer hospice stay would allow the hos- 
pice staff to implement a comprehensive care plan and allow hospices operating under daily reimbursement to be compensated for large up-front admission costs. Although hospice is not appropriate for every terminally ill patient, it seems clear that physicians are not utilizing hospice to its fullest potential or at optimal times.

\section{Physicians' influence on hospice decisions}

Patients, caregivers, hospice staff and physicians themselves all recognize the role of physicians as the gatekeepers to hospice. Studies have found that over 90\% of physicians are aware of hospice (Bonham et al. 1986; Gochman \& Bonham 1988). The majority of patients (from $63 \%$ to $83 \%$ ) whose physicians present the hospice option to them choose this alternative (Gochman \& Bonham 1988; Weggel 1999). Studies have, however, found that at least one-quarter of physicians do not discuss hospice options with their terminally ill patients, and the other three-quarters discuss hospice selectively (Gochman \& Bonham 1988; McNeilly \& Hillary 1997). In Weggel's (1999) follow-up interview, physicians cited their tendency to delay the discussion of hospice options until they thought their patients were prepared to accept such options. Prigerson's (1991) finding that $85 \%$ of patients expressed preferences for palliative care rather than curative treatment suggests that patients may be more receptive to hospice than physicians and caregivers expect.

Specialists in cardiology, gastroenterology, neurology, nephrology, oncology, pulmonology and urology were responsible for the majority of hospice recommendations in Weggel's (1999) study because primary care physicians often refer terminally ill patients to specialists for further treatment and testing. However, Christakis (1994) found that length of stay in hospice was not influenced by whether patients were referred by general practitioners or specialists. Because some research suggests that primary care physicians have greater levels of awareness of hospice care than specialists (Gochman \& Bonham 1988; but see Christakis 1994) and are more willing to discuss hospice with patients, future studies should continue to sample both primary care physicians and specialists but keep track of their designations in order to detect any differing conceptions or practices. Many of the physicians in Weggel's study suggested that nurses are often the ones who suggest to physicians that patients should be offered hospice care. Thus, future studies should also sample nurses to determine the relationship between the time when nurses first mention hospice and when physicians discuss hospice with their patients.

Regardless of which medical professional is the first to mention hospice, the timing of the hospice discussion seems to be the single most influential factor in utilization. Bonham et al. (1986) found that families who were provided hospice information before and during their relative's terminal illness were seven times more likely to consider hospice than those families who knew of hospice before the illness but did not receive further information during the illness. Although $40 \%$ of cancer patients and $68 \%$ of their families had heard of hospice before their physicians mentioned the option, physicians $(80 \%)$ were more likely than caregivers $(54 \%)$ and patients $(42 \%)$ to view themselves as the initiator of hospice discussions. Unless physicians provided hospice information following the terminal diagnosis, few patients and their families considered this option. In addition, Prigerson (1991) found that patients whose physicians told them they were dying were seven times more likely to use hospice services than patients whose physicians did not disclose their terminality. The only patient variable that affected hospice use was the patient's acceptance of death, whereas the patient's preference for alleviation of pain and suffering over prolongation of life did not increase chances of hospice utilization (Prigerson 1991). Therefore, the physician's disclosure of a terminal prognosis has both an indirect effect, through helping the patient face his or her impending death, and a direct effect on hospice use.

\section{Reasons for choosing hospice}

Both physicians and caregivers cite help with aspects of care (e.g. technical assistance with nursing and medical care), manner of dying (i.e. satisfying patients' desires to die at home), and psychological, emotional and social support as reasons for utilizing hospice services (e.g. Bonham et al. 1986). As expected, physicians who have major involvement in patient care, treat more terminally ill patients and have primary responsibility for disclosing terminality are more likely to discuss hos- 
pice (Gochman \& Bonham 1988; McNeilly \& Hillary 1997). Physicians' perceptions of hospice and the value they place on staying home, along with their ability to accept terminal diagnoses and communicate them to patients, also surface as themes to hospice decision making (Hyman \& Bulkin 1991).

McNeilly \& Hillary (1997) found that the majority of physicians cited level of help given to patients as the most important hospice service, followed by support services and manner of dying. Other studies have found that the majority of physicians cited psychological, social and emotional support services as the most important hospice services, followed by help with specific aspects of care and manner of dying (Bonham et al. 1986; Gochman \& Bonham 1988). Caregivers, on the other hand, cite manner of dying as the most important service and place less importance on the psychological, social and emotional support provided by hospice (Bonham et al. 1986; Gochman \& Bonham 1988; McNeilly \& Hillary 1997). Future research should examine whether the disparity between the reasons physicians and caregivers give for choosing hospice decreases effective communication and acceptance of hospice recommendations.

\section{Barriers to hospice utilization}

Several studies with slight variations in their methodologies have explored barriers to hospice utilization by surveying or interviewing physicians, caregivers, hospice and home health care staff, and hospice and home health care patient family survivors (Hyman \& Bulkin 1991; Prigerson 1991; McNeilly \& Hillary 1997; Weggel 1999). These studies have investigated three sources of potential barriers to hospice utilization: patient barriers, hospice structure, and physician barriers (see Table 1).

Patient barriers, such as a patient's denial of terminality and desire to continue active treatment, were explored from the perspective of physicians and primary caregivers. Physicians also discussed aspects of the hospice structure, such as the requirement of a live-in caregiver, that often exclude populations of terminally ill patients who may benefit the most from hospice services. Lastly, hospice staff, primary caregivers and physicians reported potential bar- riers created by the gatekeepers of hospice services, physicians themselves. Before focusing on physician barriers to hospice use, the most influential patient and hospice structure barriers will be discussed briefly, not only because physicians recognize their importance, but also because they contribute indirectly to the physician barriers.

\section{Patient and hospice barriers}

In Prigerson's (1991) study, physicians and primary caregivers cited the patient's lack of knowledge about hospice as the primary patient barrier to hospice use. Even when patients did know about hospice, some physicians (18\%) and primary caregivers $(26 \%)$ claimed that they did not think the patient would consent to hospice. Hyman \& Bulkin (1991) found that physicians cited the following patient-related factors as disincentives for referring patients to hospice: the patient believes that hospice means death, the patient is emotionally fragile, the patient denies his or her terminal status, and the patient wishes to continue active therapy. McNeilly \& Hillary (1997) found that the hospice staff (47\%), home health care staff (20\%), hospice patient family survivors $(2 \%)$, and home health care patient family survivors $(5 \%)$, all, to some extent, recognized the patient or family's denial of the terminal diagnosis as a barrier to choosing hospice care.

Similarly, Weggel (1999) found that physicians reported encountering four main barriers related to patients' and/or families' dispositions. Nearly half of the physicians reported that (i) the patient or family's denial of the terminal diagnosis (49\%) and (ii) the patient or family's desire to continue life-prolonging treatment (48\%) were barriers to their initiation of discussions about hospice options. Similar to Hyman \& Bulkin's (1991) finding that physicians reported the patient's emotional frailty as a barrier, the physicians in Weggel's (1999) study also cited their worries that (iii) the offer of hospice might cause the patient or family anxiety or a loss of hope (19\%). In addition (iv) the patient's wish that his or her physician make all the medical decisions (14\%) was reported as a barrier to the hospice discussion (Weggel 1999).

Physicians not only have to help patients overcome their reservations toward hospice, but they also have 
Table 1 Barriers to hospice utilization

\begin{tabular}{ll}
\hline Barriers & Examples \\
\hline Patient & $\begin{array}{c}\text { Lacks knowledge about hospice, denies terminal status, wishes to continue active } \\
\text { treatment, believes hospice means death } \\
\text { Complicated admission criteria, drug restrictions, primary caregiver requirement, } \\
\text { prognosis of } 6 \text { months or less }\end{array}$ \\
$\begin{array}{l}\text { Physician } \\
\text { Negative perceptions }\end{array}$ & $\begin{array}{c}\text { Perceives hospice as inflexible, views hospice as last resort, lacks knowledge about } \\
\text { and experience with hospice } \\
\text { Reluctant to give terminal diagnosis and prognosis, fears being blamed for giving up, } \\
\text { uncomfortable talking about death }\end{array}$ \\
Instrumentalist perspective & $\begin{array}{c}\text { Fears losing control of patient, prefers active treatment to palliative care, feels } \\
\text { pressure from cure-oriented profession } \\
\text { Overestimates life expectancy, wary of suggesting hospice, waits until patient has } \\
\text { no other options or is too fragile to transfer }\end{array}$ \\
\hline
\end{tabular}

to work around barriers built into the hospice structure. In McNeilly \& Hillary's (1997) study, the hospice staff cited the following structural problems as barriers to the hospice discussion: physicians' difficulties with hospice admission criteria due to their lack of education on procedures, drug restrictions, family structural dynamics and configurations, outdated brochures, inadequate community education programs, and insufficient hospice program marketing. Forty per cent of the physicians in Weggel's (1999) study cited the patient's having no family or friends to help provide hospice care as a barrier, compared with only $7 \%$ of the physicians and $14 \%$ of the caregivers in Prigerson's (1991) study. Physicians also reported the patient's not fitting hospice criteria (21\%) and their concern that the patient did not have adequate insurance coverage for hospice (12\%) as barriers to discussing hospice (Weggel 1999).

Hospices' stipulation that patients are not appropriate for care until they have less than 6 months to live is perhaps the most difficult structural barrier to overcome. Physicians cited their difficulty in prognosticating time to live $(59 \%)$ as the most frequent barrier to discussing hospice options (Weggel 1999). Accordingly, Hyman \& Bulkin (1991) found that physicians cited the patient's functioning being too high or too low, the patient's survival time being very limited, and the patient's disease course being difficult to predict as disincentives for referring patients to hospice.
Although physicians have little control over certain patient barriers, such as a patient's denial of terminality and desire to continue active treatment, they can nonetheless increase a patient's awareness of hospice and dispel a patient's misperceptions of hospice. Limited by current knowledge and technology, physicians also have little control over the imprecise nature of terminal prognoses. Hospices can, however, make a concerted effort to educate physicians on admission criteria and solicit suggestions for amendments to present regulations they think deny hospice to those who might benefit most from its services.

\section{Physician barriers}

Physicians can also examine the barriers to hospice use that stem from their own perceptions and priorities. We have divided physician barriers into the following categories representing the most common and detrimental barriers to hospice utilization: lack of knowledge and negative perceptions of hospice, discomfort communicating poor prognoses, instrumentalist perspective, and timing of hospice discussions (see Table 1).

\section{Lack of knowledge and negative perceptions of hospice}

As cited by hospice staff, one major barrier to hospice discussions and referrals is lack of physician experience with and awareness of hospice and its benefits 
(Hyman \& Bulkin 1991; McNeilly \& Hillary 1997). Fifteen per cent of physicians cite their lack of knowledge about local hospice programs (Weggel 1999), and $4 \%$ of physicians cite their lack of knowledge about hospice in general (Prigerson 1991), as barriers to their discussion of this option. McNeilly \& Hillary (1997) found that only $24 \%$ of physicians had personal experience working with hospice, while $28 \%$ of physicians were aware of the services and competence of hospice personnel, and $26 \%$ were simply aware of the concept of hospice.

In contrast to the fairly objective structural barriers to hospice use cited earlier, Hyman \& Bulkin (1991) found that some physicians' perceptions of hospice structures affect their decision to discuss hospice options with patients. American physicians reported that the inflexibility of hospice, exemplified by the 6-month prognosis and the outpatient-to-inpatient care ratio, delayed hospice discussion. Some physicians reported that hospice overburdens the caregiver $(16 \%)$, does not adequately delay the patient's death $(7 \%)$, and is poor at pain control (3\%) (Prigerson 1991). According to Skelly (1994), the interdisciplinary approach of hospice can also be a barrier to physicians' acceptance of its use because physicians are often reluctant to share control of patient care with non-physicians. However, hospice care plans are dependent upon the physician's approval and hospice welcomes the physician's input on care. Hospice is a means to empower patients with choices affecting end-of-life treatment; it is not a method to disable physicians' power as caregivers (Bulkin \& Lukashok 1988; Thalhuber 1995).

Bonham et al. (1986) found that most physicians and primary caregivers view hospice as an alternative, rather than as an addition, to traditional medical care. Hyman \& Bulkin (1991) found that physicians cited perceptions of hospice as 'a last resort' and 'a place where one goes to die' as disincentives to referring patients to hospice. Physicians also cited their perception that hospice care is no different from standard terminal care as a disincentive to referring patients to hospice (Hyman \& Bulkin 1991). Instead of viewing hospice as a reduction or cessation of treatment, physicians should be encouraged to recognize the role of hospice as an active component of the continuum of care (Thalhuber 1995).

\section{Discomfort communicating poor prognoses}

The most common reason for not admitting patients to hospice is their death before hospice care could be arranged (MacDonald 1989). Several factors may contribute to this situation: the reluctance of society (especially American) to accept the dying process as natural, the difficulty of accurately predicting death, and the hesitancy of physicians to discontinue curative treatment in favor of hospice. Not only are physicians continuing active treatment, $25 \%$ of them report that they have not engaged in any hospice discussions with any of their terminally ill patients within the last year (Gochman \& Bonham 1988; McNeilly \& Hillary 1997). Physicians may be avoiding or delaying hospice discussions due to their difficulty accepting and discussing terminal diagnoses and prognoses with patients. Two hurdles must be overcome before a physician even broaches the subject of hospice with a patient: first, the physician has to accept the terminality of the patient's illness, and secondly, the physician has to communicate that terminality to the patient.

The majority of the hospice staff in McNeilly \& Hillary's (1997) study cited several problems involving physicians as barriers to the discussion of hospice options: deficient physician explanations of terminal illness to patients, insufficient physician education about palliative care, and a lack of physician comfort with death and the hospice concept. According to the hospice staff, physicians' lack of comfort with death stems from their difficulty admitting their limitations when dealing with terminal illness. Physicians may be reluctant to discuss patients' prognoses, fearing they will be blamed for poor outcomes (Schonwetter et al. 1990). Gordon (1989) claimed that physicians often do not refer patients to hospice due to their fear that it would diminish their stature in the eyes of their patients and families and due to their own inability to accept the inevitable death of their patients.

McNeilly \& Hillary (1997) found that $37 \%$ of physicians felt that the hospice discussion was manageable and generally straightforward, $17 \%$ felt the discussion was uncomfortable but manageable, and $11 \%$ simply felt discomfort. Consistent with other studies (Hyman \& Bulkin 1991; Prigerson 1991), Weggel (1999) found that $16 \%$ of physicians cited their difficulty telling the 
patient about the terminal diagnosis as a barrier, and $6 \%$ cited their discomfort talking about death and dying issues as barriers to hospice discussions.

\section{Instrumentalist perspective}

The hospice staff also cited physicians' fear of losing control of their patients as a barrier to hospice utilization, especially when long-term relationships had developed (Hyman \& Bulkin 1991; McNeilly \& Hillary 1997). Hyman \& Bulkin (1991) cited physicians' perception of hospice as 'doing nothing rather than something' as a disincentive for referring patients to hospice. If physicians perceive hospice as doing nothing, they may not refer patients, seeing little reason to take steps toward transferring care. Similarly, Weggel (1999) found that physicians in the follow-up interview expressed concerns about lost contact and involvement with patients. Physicians with this kind of 'instrumentalist' perspective prefer using scientific advancements actively to disrupt, rather than passively to accept, the disease course. Prigerson (1991) predicted that physicians' lack of personal experience with death, affiliation with a teaching hospital, and fear of malpractice would predispose physicians to be instrumentalist in their approach towards terminal care. He found that affiliation with a teaching hospital and fear of malpractice indeed both reduced hospice utilization, whereas lack of personal experience with death did not have an effect.

Along the same lines, Weggel (1999) found that some physicians cited the medical community's expectation of cure-oriented treatment (4\%) and the feeling of professional defeat upon suggesting hospice $(2 \%)$ as barriers to hospice discussions. Some physicians are simply loath to abandon aggressive treatment, perceiving the cessation of curative measures for palliative care as failure (Hyman \& Bulkin 1991; Weggel 1999).

All these instrumentalist barriers to the hospice discussion relate to a common decision-making heuristic: sunk-cost bias. Sunk-cost bias occurs when a decision maker continues to invest resources into a previously selected action or plan even after the plan is perceived to be suboptimal. Bornstein et al. (1999) found that medical residents exhibited a sunk-cost bias in the sense that they were less likely to suggest that physicians currently treating a patient should change their management plans when that physician had initiated the plan than when another physician had. In an effort to appear consistent, physicians treating terminal patients may similarly opt to continue curative measures rather than switch to palliative care and/or transfer care to a hospice team.

\section{Timing of hospice discussions}

The combination of physicians' difficulty broaching the subject of hospice and their instrumentalist perspective has led to a well-recognized barrier to hospice utilization, the timing of hospice discussions. Physicians recognize their tendency to suggest hospice too late in the disease course (Weggel 1999). Because physicians commonly overestimate the life expectancy of their terminally ill patients, they do not bring up the subject of hospice care until it has become too stressful for patients to be transferred (Rhymes 1990). McNeilly \& Hillary (1997) found that a substantial number of physicians (28\%) did not perceive hospice discussions as appropriate until treatment was no longer effective and the patient entered a deteriorating state, whereas $24 \%$ perceived the time when the terminal diagnosis was first made as the appropriate time. Many physicians considered patients appropriate for hospice care when they had accepted their terminal illness (41\%), as opposed to meeting the 'less than 6 months to live' hospice criterion (26\%) (McNeilly \& Hillary 1997).

According to Hyman \& Bulkin (1991), the patient's and physician's acceptance of the terminal diagnosis, along with the 6-month hospice regulation, determined when the physician discussed hospice with the patient. Some physicians prepared patients early in the disease course for later hospice options, whereas others waited until the patients had no other options. Regardless, the majority of hospice family survivors (39\%) reported that they would have welcomed more information about hospice from their physicians at the time the diagnosis was labelled terminal, and the majority of home health care family survivors $(50 \%)$ would have welcomed more information at the time of diagnosis (McNeilly \& Hillary 1997). Based on this finding, physicians should be encouraged to raise the subject of hospice early in the terminal disease course, so that patients can decide for themselves if and when the hospice option is right for them. 


\section{The next step}

Although the studies discussed above provide useful insights into the barriers that hinder hospice utilization, they are somewhat simplistic. As surveys, the studies rely on self-reports from participants, but people's introspective reports about the causes of their behaviors and cognitive processes are often inaccurate (Nisbett \& Wilson 1977; Wilson \& Nisbett 1978). In order to gain a more comprehensive understanding of barriers to hospice use, systematic investigations need to be conducted. Three possible approaches to such systematic study are archival studies, observational studies and experimental simulations.

Through archival studies, researchers could retrospectively code which patients were referred to hospice and determine what patient and physician factors predict referral. Ideally, researchers could prospectively observe practicing physicians, comparing patient and physician factors that lead to hospice referrals and those that do not. Although both of these study designs would provide realistic data, archival studies are limited to the information previously collected and observational studies are logistically complicated. In both types of studies, it is also hard to control for all possible confounds and code attitudes and behaviors appropriately.

If researchers want to conduct easier and more carefully controlled experiments, they could simulate situations that involve hospice decisions and sample a range of participants from students to specialists. Simulations have been used to study the fundamental psychological processes underlying real-world decisions in a number of domains, including medical decision making (e.g. Bornstein et al. 1999). Unlike past studies that have only asked physicians what factors they consider barriers to the hospice discussion, future research could use patient vignettes to discern whether factors physicians claim are barriers actually affect their decision making. For example, researchers could manipulate factors related to perceived barriers, such as timing or the energy already invested in a patient, in patient vignettes to determine their effects on participants' decisions. Future research could systematically determine when physicians consider hospice discussions appropriate by presenting physicians with patient vignettes and asking them how likely they would be to mention hos- pice at critical points in the disease course. In general, researchers should use different study designs, such as the archival, observation and simulation methods, to gather a comprehensive understanding of barriers to hospice utilization.

Physicians can also strive to increase hospice utilization by realizing their own discomfort in dealing with terminal patients and by exploring ways they can better serve these patients. Physicians taking proactive stances towards disclosing terminal diagnoses and perceiving hospice as death with dignity are crucial to effective hospice use (Hyman \& Bulkin 1991). Physician education about hospice is critical to its increased utilization. Training in pain management and palliative care during medical school would increase the acceptance of hospice as part of the continuum of care, teaching physicians how to care for those they cannot cure (Bulkin \& Lukashok 1988; Thalhuber 1995). Thereafter, physicians should be periodically taught methods for dealing with dying patients and reminded of the procedures for transferring patients to hospice. Hospice staff cite follow-up instruction and referral education procedures for physicians who have made late patient referrals as structural facilitators to hospice utilization (McNeilly \& Hillary 1997). In addition to listening and addressing physicians' concerns, hospice staff need to take the time to explain the intricacies of hospice, especially reimbursement, to physicians (Thalhuber 1995). Because hospice patients are being referred by physicians with scientific orientations, systematic clinical data on hospice should be collected and conveyed to the medical community (Thalhuber 1995).

\section{Conclusions}

Research into the barriers that hinder hospice care will hopefully lead to a better understanding of endof-life decisions in general, and to greater utilization of the comprehensive and cost-effective hospice option specifically. Rather than viewing hospice as foreign or something to be feared, society should be able to view hospice as a comfortable part of the continuum of comprehensive care (Zuckerman \& Wollner 1999). Generally, physicians are skilled at dealing with endof-life care. It is not our intention to be critical of phy- 
sicians' care but merely to suggest ways that they can better utilize hospice services.

Gochman \& Bonham (1988) speculated that four factors limit physicians' use of hospice: (i) physicians do not agree with the hospice concept or appreciate the services hospice provides; (ii) they believe care provided by them and others is more appropriate than hospice care; (iii) they do not routinely think about hospice; or (iv) they have difficulty accepting and discussing terminal illness with patients.

Studies do not support Gochman \& Bonham's (1988) first speculation that physicians do not agree with the hospice concept or appreciate the services hospice provides. The majority of physicians are positive about and open to resources that would be helpful in discussing terminality and hospice options (McNeilly \& Hillary 1997). The discrepancy between how many physicians know about hospice and how many actually discuss it also cannot be explained by physicians' negative experiences with hospice or patients' rejections of the suggestion.

Regarding Gochman \& Bonham's (1988) second speculation, Hyman \& Bulkin (1991) found that physicians did cite their comfort with and expertise in palliative care as a reason to keep terminal patients under their care, favoring their care rather than hospice care. When physicians are experienced in terminal care, transfer to hospice may be more disruptive than beneficial to patients. However, given the high percentage of physicians who are not comfortable discussing terminal issues and who have an instrumentalist perspective, the percentage of physicians choosing not to use hospice due to their high degree of comfort with palliative care is probably rather small.

Although research has investigated when and why physicians discuss hospice, little research has explored Gochman and Bonham's third speculation, that physicians do not routinely think about hospice. Studies have found that over $90 \%$ of physicians are aware of hospice when specifically asked about the service (Bonham et al. 1986; Gochman \& Bonham 1988). This figure shows that thoughts of hospice are available to most physicians but does not provide information about the accessibility of such thoughts. Higgins (1996) reviewed a body of research to demonstrate that use of available knowledge increases as accessibility to that knowledge increases. The discrepancy between the availability and accessibility of hospice knowledge could explain why most physicians are aware of hospice, but at least one-quarter of physicians do not discuss hospice options with their terminally ill patients and the other three-quarters discuss hospice selectively (Gochman \& Bonham 1988; McNeilly \& Hillary 1997). Perhaps physicians are not discussing hospice with patients simply because the option does not come readily to mind. Research needs to be conducted to explore this speculation.

Based on past research, another reason physicians do not use hospice is Gochman \& Bonham's (1988) fourth speculation, that physicians have difficulty accepting and discussing terminal illness with patients. Hospice staff cite physicians' lack of comfort with death and the hospice concept and their fears of losing control of patients as major barriers to the initiation of the hospice discussion (McNeilly \& Hillary 1997). Physicians recognize their own difficulties discussing terminal diagnoses and death along with the pressure they feel from the medical community to provide cure-oriented treatment (Weggel 1999). Due to this discomfort, physicians recognize their tendency to suggest hospice too late in the disease course, when patients are no longer able to benefit from hospice care (Weggel 1999).

Based on the studies reviewed, several factors seem essential to hospice utilization. Hospice is most likely to be discussed and chosen when: (i) patients acknowledge their impending death and prefer palliative to curative measures; (ii) patients meet the requirements of hospice admission; (iii) their physicians understand the boundaries and flexibility of these requirements; and (iv) physicians recognize the benefits of palliative care and do not view transfers to hospice as failures or cessation of their care. Because physicians are the gatekeepers to hospice, their discomfort discussing death and terminal care with dying patients is a major obstacle to hospice utilization. Physicians need to realize their considerable influence in either facilitating or hindering hospice utilization.

\section{Acknowledgements}

The authors thank Christie Emler for helpful comments on an earlier version of the manuscript. 


\section{References}

Bonham G.S., Gochman D.S., Burgess L. \& Fream A.M. (1986) The Hospice Decision: Multiple Determinants. University of Louisville Urban Studies Center, Louisville, KY.

Bornstein B.H., Emler A.C. \& Chapman G.B. (1999) Rationality in medical treatment decisions: is there a sunk-cost bias effect? Social Science and Medicine 29, 215-222.

Bulkin W. \& Lukashok H. (1988) Rx for dying: the case for hospice. New England Journal of Medicine 318, 376-378.

Carwein V.L. (1986) Home hospice care in Britain and the United States. Caring 5, 54-57.

Christakis N.A. (1994) Timing of referral of terminally ill patients to an outpatient hospice. Journal of General Internal Medicine 9, 314-320.

Christakis N.A. (1997) Survival of Medicare patients enrolled in hospice programs. Presentation at the annual American Academy of Hospice and Palliative Medicine meeting, Chicago, 21-25 June 1997.

Christakis N.A. \& Escarce J.J. (1996) Survival of Medicare patients after enrollment in hospice programs. New England Journal of Medicine 335, 172-178.

Emanuel E.J. (1996) Cost savings at the end of life: what do the data show? Journal of the American Medical Association 275, 1907-1914.

Emanuel E.J., Ash A., Yu W., Gazelle G., Levinsky N.G., Saynina O., McClellan M. \& Moskowitz M. (2002) Managed care, hospice use, site of death, and medical expenditures in the last year of life. Archives of Internal Medicine 162, 1722-1728.

Gochman D.S. \& Bonham G.S. (1988) Physicians and the hospice decision: awareness, discussion, reasons, and satisfaction. Hospice Journal 4 (1), 25-53.

Gordon A.K. (1989) The physician gatekeeper: access to the Medicare hospice benefit. American Journal of Hospice Care 6 (5), 44-47.

Higgins E.T. (1996) Knowledge activation: accessibility, applicability, and salience. In Social Psychology: Handbook of Basic Principles (eds. T.E. Higgins \& A.W. Kruglanski), pp. 133-168. Guilford Press, New York.

Hospice Information Service (2002) Palliative care facts and figures [WWW document]. URL http://www.hospiceinformation.info/factsandfigures/services.asp
Hyman R.B. \& Bulkin W. (1991) Physician reported incentives and disincentives for referring patients to hospice. Hospice Journal 6 (4), 39-64.

MacDonald D. (1989) Non-admissions: the other side of the hospice story. American Journal of Hospice Care 6 (2), 17-19, 40-42.

McNeilly D.P. \& Hillary K. (1997) The hospice decision: psychological facilitators and barriers. Omega 35, 193-217.

National Statistics on Death and Hospice (1994) National statistics on death and hospice. Thanatos 19 (3), 35.

Nisbett R.E. \& Wilson T.D. (1977) Telling more than we can know: verbal reports on mental processes. Psychological Review 84, 231-259.

Pearlman R.A. (1988) Inaccurate predictions of life expectancy: dilemmas and opportunities. Archive of Internal Medicine 148, 2537-2538.

Prigerson H.G. (1991) Determinants of hospice utilization among terminally ill geriatric patients. Home Health Care Services Quarterly 12 (4), 81-112.

Rhymes J. (1990) Hospice care in America. Journal of the American Medical Association 264, 369-372.

Schonwetter R.S., Teasdale T.A., Storey P. \& Luchi R.J. (1990) Estimation of survival time in terminal cancer patients: an impedance to hospice admissions? Hospice Journal 6 (4), 65-79.

Skelly F.J. (1994) Hospice. American Medical News 37 (11), 15-19.

Stillman M.J. \& Syrjala K.L. (1999) Differences in physician access patterns to hospice care. Journal of Pain and Symptom Management 17, 157-163.

Thalhuber W.H. (1995) Overcoming physician barriers to hospice care. Minnesota Medicine 78, 18-22.

Weggel J.M. (1999) Barriers to the physician decision to offer hospice as an option for terminal care. Wisconsin Medical Journal 98 (3), 49-53.

Wilson T.D. \& Nisbett R.E. (1978) The accuracy of verbal reports about the effects of stimuli on evaluations and behavior. Social Psychology 41, 118-131.

Zuckerman C. \& Wollner D. (1999) End of life care and decision making: how far we have come, how far we have to go. Hospice Journal 14 (3-4), 85-107. 